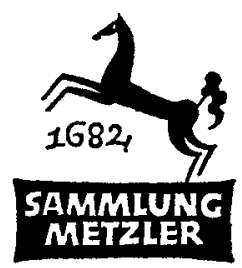

REALIENBÜCHER FÚR GERMANISTEN ABT. C:

DEUTSCHE SPRACHWISSENSCHAFT 
HANS GLINZ

\section{Deutsche Syntax}

\section{MCMLXV}

J. B. METZLERSCHE VERLAGSBUCHHANDLUNG STUTTGART 
ISBN 978-3-476-99102-7

ISBN 978-3-476-99101-0 (eBook)

DOI 10.1007/978-3-476-99101-0

(C) 1965 Springer-Verlag GmbH Deutschland

Ursprünglich erschienen bei J. B. Metzlersehe Verlagsbuchhandlung und Carl Ernst Poeschel Verlag GmbH in Stuttgart 1965 


\section{INHALT}

Wichtige Literatur . . . . . . . . . . . . . . . VII

I. Stellung und Aufgabe der Syntax Im Rahmen der MEISTEN GRAMMATIKEN

1. Schultradition und Lexika . . . . . . . . . . 1

2. Germanistische Elementarbuicher . . . . . . . 2

3. Große Grammatiken deutscher Germanisten . . . . 3

4. Internationale Linguistik; Strukturalismus . . . . . 4

II. Die Steliung von Syntax, Phonologie und MorphoLOGIE BEI UNMTT'TELBARER BEOBACHTUNG

1. Beobachtungen an der Kindersprache . . . . . . . 6

2. Beobachtungen beim Fremdsprachen-Unterricht . . 7

3. Beobachtungen beim Vorgehen empirischstruktureller Sprachbeschreibung . . . . . . . . . 8

III. Sprachtheoretische Grundbegriffe

1. Grundsätzliches . . . . . . . . . . . 9 9

2. Satz . . . . . . . . . . . . 9

3. Wort . . . . . . . . . . . . . 10

4. Verhältnis der sprachlichen Inhalte („Begriffe“, „Bedeutungen", "meaning" und der sie tragenden Lautungen)

5. "Geltende Sprachinhalte" und "dienende Sprachinhalte"; Nomosphäre und Morphosphäre; subsemantische Phänomene. . . . . . . . . 12

6. Lautungsunterschiede ohne inhaltliche Relevanz; Phonomorphie . . . . . . . . . . . 14

7. Die Klangseite der Sprache, im Ganzen betrachtet . . 14

8. Überblick . . . . . . . . . . . . . . 15

IV. SKizze DER AUfGabeN UND METHOden MODERNER SYNTAKTISCHER FORSCHUNG

1. Aufgaben . . . . . . . . . . . .

2. Methoden . . . . . . . . . . . . .

3. Sprachzustand und Sprachgeschichte; Synchronie und Diachronie . . . . . . . . . 20

V. Bekannte Darstellungen DeR DeUtschen Syntax 1837-1932

1. Jacob Grimm (1785-1863) . . . . . . . . 21

2. Oskar Erdmann (1846-1895). . . . . . . . . 25

3. Hermann Wunderlich $(1858-1916) \ldots . . . .26$ 
4. Wilhelm Wilmanns (1842-1911) . . . . . . 32

5. Hermann Paul (1846-1921) . . . . . . . . 34

6. George O. Curme (1860-1948) . . . . . . . 41

7. Hans Reis (1867-1933) . . . . . . . . . 43

8. Otto Behaghel (1854-1936) . . . . . . . . 47

9. Wilhelm Havers (1879-1961) . . . . . . . 53

10. Zwischenbilanz $1837^{-1932}$......... 54

VI. ENTWICKLUNG NEUER SYNTAKTISCHER BEgRIFFE UND METHODEN

1. Zwei Urteile über die Situation in den zoer und $40 \mathrm{er}$ Jahren .............. 55

2. Skizze der Entwicklung seit den 2oer und 3oer Jahren 57

3. Zusammenwirken von Wissenschaft und Schulpraxis 60

VII. VERSUChe EINER INHALTBEZOGENEN DEUTSCHEN SYNTAX 1958-1963

1. Johannes Erben (geb. 1925) . . . . . . . . 63

2. Paul Grebe (geb. 1908) . . . . . . . . . . . . 67

3. Hennig Brinkmann (geb. 1901) . . . . . . 72

4. Leo Weisgerber (geb. 1899) . . . . . . . . . . 81

VIII. Deutsche Syntax als "Generative grammar"

1. Allgemeines . . . . . . . . . . . . . . . . . . 89

2. Deskriptive amerikanische Linguistik 1940-1960 . . 90

3. Noam Chomsky und seine generative grammar . . . 92

4. Arbeitsstelle strukturelle Grammatik, Berlin . . . . 98

IX. Aufgaben Für KOMMENde ARbeit aN DER DeUtSChen Syntax

1. Unterscheidung der Arbeitsbereiche und Entwicklung der Methoden . . . . . . . . . . . 101

2. Möglichkeiten für den Aufbau einer Darstellung . . 103

3. Ausdehnung auf ältere Sprachstufen; Problem der Geschichte übethaupt . . . . . . . 105

RegISter .............. 109 


\section{Wichtige Literatur}

Auf ein Zusammentragen aller erreichbaren Literatur wurde verzichtet, da das hier nicht möglich ist und auch kaum sinnvoll erscheint; dagegen wurde eine einigermaßen repräsentative Auswahl angestrebt, die dem Suchenden weitere Wege zeigen und ebnen wird.

\section{x. Darstellungen im Rabmen von Gesamt-Grammatiken}

DUDEN, Grammatik der deutschen Gegenwartssprache $(=$ Der große Duden, Bd 4), hrsg. v. d. Dudenredaktion unter Leitung von PAUl GreBe, unter Mitwirkung namhafter Fachgelehrter. Völlig neubearb. Ausgabe, 1959.

Johannes Erben, Abriß der deutschen Grammatik, 1958, 71964.

Jean Fourquet, Grammaire de l'Allemand, Paris 195 2, ${ }^{2} 1956$.

George O. Curme, A Grammar of the German Language. New York 1904; 2d rev. Ed., New York 1922, ${ }^{1} 1952$.

Hermann Paul, Deutsche Grammatik, 5 Bde, 1916/20, 51959. (Die Syntax in Bd 3 u. 4.)

Wilhelm Wilmanns, Deutsche Grammatik, Gotisch, Alt-, Mittelund Neuhochdeutsch, Straßburg 11893-1897, 21896-1909 (Syntaktisches in Band 3/1 und 3/2).

Jacoв Grimm, Deutsche Grammatik, 4 Bde, 1837 (die Syntax in Bd 4); neuer, vermehrter Abdruck durch Gustav Rozthe, 1898.

\section{Selbständig erscbienene Darstellungen}

Otto Behaghex, Deutsche Syntax, eine geschichtliche Darstellung, 4 Bde, $1923 / 32$.

Wrlhelm Havers, Handbuch der erklärenden Syntax, 1931.

Hermann Wunderlich und Hans Reis, Der deutsche Satzbau: 1. Aufl. in $1 \mathrm{Bd}, 1892$; 2. Aufl. 1901,2 Bde, von HermanN WunderLICH; 3. Aufl. 1924, 2 Bde, bearb. von Hans ReIs.

Oskar Erdmann, Grundzüge der deutschen Syntax, 1886; Bd 2, bearb. von Otto Mensing, 1897.

\section{Monograpbien und Untersucbungen zur Begriffsbildung und Methode}

Hans Glinz, Grundbegriffe und Methoden inhaltbezogener Text- $u$. Sprachanalyse, 1965 .

Manfred Bierwisch, Grammatik des deutschen Verbs, 1963. (Studia Grammatica. II.) 
Leo Wersgerber, Die vier Stufen in der Erforschung der Sprachen, 1963. (Sprache und Gemeinschaft, Grundlegung, Bd. II.) - zit. : Vier Stufen.

Hennig Brinkmann, Die deutsche Sprache. Gestalt u. Leistung, 1962. (Sprache und Gemeinschaft, Grundlegung, Bd I.)

Hans Glinz, Ansätze zu einer Sprachtheorie, 1962. (Wirkendes Wort, Beiheft 2.) - zit.: Ansätze.

Hugo Moser (Hrsg.), Das Ringen um eine neue deutsche Grammatik, Aufsätze aus drei Jahrzehnten (1929-1959), 1962. - zit.: Sammelband Moser.

Hans Glinz, Der deutsche Satz. Satzglieder u. Wortarten wissenschaftlich gefaßt u. dichterisch gedeutet. 1957, ${ }^{41965}$. - zit.: Deutscber Satz.

Charles C. Fries, The Structure of English, An Introduction to the Construction of English Sentences, New York $1952,{ }^{4} 1963$.

Hans Glinz, Die innere Form des Deutschen, Eine neue deutsche Grammatik, 1952, ${ }^{4}$ 1964. - zit.: Innere Form.

Alexander Rosetti, Le mot. Esquisse d'une théorie générale. Copenhague et Bucuresti 1943. (Mémoires de la Société Roumaine de linguistique. Série I, 3.)

Fritz Rahn und Wolfgang Pfleiderer, Neue Satzlehre, 1940.

Jean Fourquet, L'ordre des eléments de la phrase en Germanique ancien, étude de syntaxe de position, Strasbourg 1938.

Erich Drach, Grundgedanken der deutschen Satzlehre, 1937.

Julius Stenzel, Philosophie der Sprache, 1934 (in: Handbuch der Philosophie).

LeOnard Bloomfield, Language, New York 1933, letzte Aufl. 1963. Alan H. Gardiner, The Theory of Speech and Language, Oxford 1932.

Leo WeIsGerber, Muttersprache und Geistesbildung, 1929.

John Ries, Beiträge zur Grundlage der Syntax: Was ist Syntax? ${ }^{1}$ Marburg 1894, 'Prag 1927; Zur Wortgruppenlehre, Prag 1928; Was ist ein Satz? Prag 1931.

Ferdinand de Saussure, Cours de linguistique générale, Paris 1916; seither viele Neudrucke-zit. : Cours.

\section{Zur älteren Gescbicbte wichtiger syntaktischer Begriffe}

Hans Glinz, Geschichte und Kritik der Lehre von den Satzgliedern in der deutschen Grammatik, 1947. - zit.: Gescbichte und Kritik.

Eugen SEIDEL, Geschichte und Kritik der wichtigsten Satzdefinitionen, 1935 .

Max Hermann Jelinek, Geschichte der neuhochdeutschen Grammatik von den Anfängen bis auf Adelung, 2 Bde, 1913/14.

August Engelien, Geschichte der neuhochdeutschen Grammatik, in: KARL KeHR, Geschichte der Methodik des deutschen Volksschulunterrichts, ${ }^{2}+889$. 


\section{Verbandlungen der Internationalen Linguisten-Kongresse}

Actes du Sixième Congrès International des Linguistes (Paris 1948), publiés sous le patronage du C.I.P.L. avec le concours de l' U.N.E.S-C.O. par les soins de M. Mrchel Lejeune, Paris 1949.

Proceedings of the Seventh International Congress of Linguists (London 1952), published under the ausspices of C.I.P.L. with the assistance of U.N.E.S.C.O. by F. Norman and P. F. GANZ, London 1956. - zit. : Proceedings I952.

Proceedings of the Eighth International Congress of Linguists (Oslo 1957). General Editor: Eva Sivertsen, Oslo 1958. - zit: Proceedings 1957 .

Proceedings of the Ninth International Congress of Linguists (Cambridge/Mass. 1962), ed. by Horace G. Lunt, Den Haag/London/ Paris 1964. - zit.: Proceedings 1962.

\section{Darstellung in Scbulbücbern auf moderner wissenscbaftlicher Grundlage}

a) für den Deutschunterricht an Anderssprachige:

Dora Schulz und Heinz Griesbach, Deutsche Sprachlehre für Ausländer. 14., methodisch neubearb. Aufl., 1962.

b) für Deutsch als Muttersprache:

Arnold/Glinz/Zimmermann, Deutscher Sprachspiegel. Sprachgestaltung u. Sprachbetrachtung, H. 1-4 (für 5.-11. Klasse), 1956 bis 1964 (die Syntax jeweils in Teil IV: Einsicht in den Bau der Sprache).

Weitere Literatur ist an den betr. Stellen im Text angeführt. 